\title{
Mental disorders and sociodemographic variables in population assisted by the Family Health Program in Brazil
}

\author{
Daniel Maffasioli Gonçalves, Suzi Camey* \\ Programa de Pós-Graduação em Epidemiologia, Universidade Federal do Rio Grande do Sul, Porto Alegre, Brazil \\ Email: danielmgpoa@gmail.com
}

Received 20 July 2012; revised 23 August 2012; accepted 31 August 2012

\begin{abstract}
Objectives: This paper aims to estimate the associations between common mental disorders (CMD) as well as cases of alcohol abuse/dependence (ADD) with sociodemographic variables in population assisted by Family Health Program (FHP) in Santa Cruz do Sul, Southern Brazil. Methods: All residents over 14 years of age from 3 areas assisted by the FHP were invited to participate between 10 February 2006 to 10 February 2007. Results: Of 2921 participants, the prevalence estimates of suspected cases of CMD and ADD were $\mathbf{2 9 . 9 3 \%}$ and $\mathbf{1 2 . 0 7 \%}$, respectively. Female, unfavorable employment situation, low schooling and low income showed a positive and independent association with CMD. Male, unfavorable employment situation and CMD showed an independent association with ADD. Conclusions: Groups with unfavorable socioeconomic conditions present the highest risk and should be taken into account when planning public mental health policies.
\end{abstract}

Keywords: Health Planning; Mental Disorders; Family Health Program; Primary Health Care; Risk Groups

\section{INTRODUCTION}

Mental disorders are increasingly recognized as an important public health problem. Prevalence estimates of mental disorders in population survey studies range from $12.2 \%$ to $48.6 \%$, depending on the screening or diagnostic methods used. About $90 \%$ of these prevalence estimates are related to common mental disorders (CMD -mood, anxiety and somatization disorders) and alcohol abuse or dependence (AAD) [1].

The Global Burden of Disease study, conducted in the 1990s, showed that in individuals between 15 and 44 years of age $12.3 \%$ of the total disease burden (disa-

"Corresponding author. bility-adjusted life years) and $30.8 \%$ of years lived with disability (YLD) in the world are due to psychiatric conditions. Among the ten main causes of global burden in this age group, six are mental disorders and among the ten main causes of YLD, seven are mental disorders [1]. Despite this fact, there is an important treatment gap in mental health. It is estimated that $40 \%$ to $70 \%$ of people suffering of mental disorder do not receive adequate diagnosis and treatment [2].

In order to offer mental health support to a larger part of the population, public policies by the WHO have been recommended to take place at the primary care level [1-3]. This is important especially because $20 \%$ to $50 \%$ of patients that look for medical support at this level of care have at least one psychiatric disorder [1,3]. For the implementation of these policies, an essential aspect is to know the epidemiologic characteristics of these disorders at each local setting $[3,4]$. Epidemiologic studies conducted in Brazilian general population are needed, especially considering the continental dimensions of this country.

The predominance of biological models in literature has made the study on socioeconomic factors a secondorder issue. However, they are essential for the identification of more susceptible population segments and it is fundamental for the public health planning. Besides that, it is important for the understanding of health-disease processes when investigating and proposing risk factors to disorders [2-5].

The purpose of this study is to explore possible associations between suspected cases of CMD and suspected cases of AAD in a sample of the population assisted by teams of the Estratégia Saúde da Família (ESF or Family Health Strategy) in the City of Santa Cruz do Sul, Southern Brazil.

\section{METHODS}

\subsection{Sample}

Santa Cruz do Sul is located in the state of Rio Grande 
do Sul, Brazil, with a population of 120,000 habitants. The city has presented high coefficients of suicide in the last years. In 2005, for example, while Brazil reported coefficient of 4.64/100,000, Santa Cruz do Sul reported $16.1 / 100,000$; that is, 3.5 as much as the national average [6].

There are eight ESF teams in Santa Cruz do Sul that assist a total population of 20,551 subjects over 14 years of age. Each ESF team is responsible for the habitants of a specific region of the city. We selected by convenience three ESF areas for the study that correspond to 5365 eligible subjects.

We considered as inclusion criteria individuals over 14 years of age living in the studied areas. The only exclusion criterion was the presence of physical or mental problem that would not allow the participation.

The percentage of refusals was $41.20 \%$ (2311) and $4.34 \%$ (233) were excluded because questionnaires with missing data. There were no statistical differences between the latter and the final sample in relation to sociodemographic variables and CMD/AAD screening. The final sample was composed by 2921 subjects that correspond to $54.46 \%$ of the original sample and $14.21 \%$ of the total population assisted by ESFs in the city.

In order to verify whether the final sample had the same sociodemographic characteristics as those of the total population assisted by ESF in the city, we compared the sociodemographic variables of all ESF population taken from the SIAB (Basic Information System) records with those from the final sample. The homogeneity of proportions by chi-square test with $\mathrm{p}<0.05$ was used. We concluded that the sample had the same sociodemographic characteristics as those of the total population considered, except for gender, as there are more women in the sample.

\subsection{Instruments}

\subsubsection{Questionnaire on Demographic Data}

Self-reporting questionnaire composed of seven questions about sociodemographic data: gender, ethnicity, marital status, age, schooling level, employment situation and family income.

\subsubsection{SRQ-20 and SRQ-A}

SRQ-20 (Self-reporting Questionnaire 20 items) is a screening instrument for CMD with 20 items proposed by Harding et al [7]. SRQ-A is a screening instrument for AAD with five items proposed by Climent and De Arango [8]. The answers are yes/no type. Each affirmative answer adds 1 to the final score, which is the sum of all affirmative answers. According to the last performance assessment of SRQ-20 and SRQ-A conducted in Brazil [9], individuals with score 8 or more (cutoff point
$7 / 8)$ in the first are considered as suspected of CMD (86.33\% sensitivity and $89.31 \%$ specificity) and individuals with score 2 or more (cutoff point 1/2) in SRQ-A are considered as suspected of AAD (69\% sensitivity and $91 \%$ specificity).

\subsection{Design}

This is a cross-sectional study conducted from 10 February 2006 to 10 February 2007 that used questionaires on sociodemographic data, SRQ-20 and SRQ-A. The questionnaire application was performed by community health workers (CHWs) during the daily visits to homes covered by the program. The invitation was made more than once to each home, since the average home visitation of the ESF is approximately 0.8 monthly visits per home.

The CHWs received specific training on the application of the questionnaires and on mental health issues before the study. They received technical and emotional support during the study as well.

All eligible residents were invited and when a resident was not at home on the occasion of the visit, the invitation for the study participation was made orally through the present relative and later in writing, and when required, the visit was scheduled. The questionnaires are self-reporting and, if the interviewee was illiterate, the instruments were read by a third person, preferably the $\mathrm{CHW}$, as the SRQ aims at including all types of subject, including illiterates [7].

\subsection{Statistical Analysis}

Data compilation and analyses were performed in SPSS program, version 16.0. Mean values and standard deviations were calculated for continuous data and frequencies for categorical variables.

As mentioned above, there were more females in the final sample than in the ESF population of Santa Cruz do Sul. For this reason, all statistical analyses were weighted for gender, according to the calculation: 1 divided by the probability of the sample including a male or female individual considering the distribution of the proportions of these categories in the sample and in the population.

The first two analyses verified the associations between suspected cases of CMD and sociodemographic variables, and between the suspected cases of AAD and sociodemographic variables as well as CMD. The univariate analysis used Poisson Regression Method with robust variance and the calculation of prevalence ratio (PR) and $\mathrm{p}$ value. After that, a multivariate analysis was performed, also using Poisson Regression Method with robust variance including all variables that presented $\mathrm{p}<$ 0.2 in the univariate analysis to analyze the effect of each 
variable independently. The final model maintained only variables that showed a significant association at 5\% level, regardless of the other variables. Then, PR values were calculated for each variable adjusted to the effect of the other variables in the final model, as well as their confidence intervals of $95 \%$.

\subsection{Ethical Aspects}

This type of study presents minimum risk in accordance with the Health Research Norms of CNS (National Health Council). The study protocol was submitted to the Research Ethics Committee of the Hospital de Clínicas de Porto Alegre (process 05565) and analysis of the Department of Health of Santa Cruz do Sul. All participants filled and signed in the informed consent term.

\section{RESULTS}

The total sample $(\mathrm{n}=2921)$ is distributed in relation to the sociodemographic variables according to Table 1. We found the predominance of female, white, married, individuals between 20 and 39 years of age, individuals with incomplete fundamental schooling level, employed and individuals with income of 1 to 3 minimum wages.

Regarding the screening for CMD and AAD, we found prevalence estimates (weighted for gender) of $29.93 \%$ (IC 95\% 29.30\% - 30.56\%) and 12.07\% (IC 95\% 11.63\% - $12.52 \%$ ), respectively. When we consider the sensitivity and specificity of the screening tools, the adjusted prevalence estimates are $21.33 \%$ for CMD and $5 \%$ for AAD [4].

There was significant association of all sociodemographic variables with CMD in the univariate analysis (Table 2); so all of them were included in the first stage of the multivariate analysis. In this step, ethnicity did not show significance and therefore was not considered in the second stage.

As we can see on Table 2, the same standard of association with CMD observed in the univariate analysis for gender, employment, schooling and income was observed in the multivariate; that is, higher PRs of CMD for women, unfavorable employment situation, low schooling level and low income. On the other hand, the age categories (40 - 49 and 50 - 49 years) that were significantly associated with higher prevalence of CMD in the univariate analysis did not show significance in the multivariate analysis. Only the category over 60 years of age, which did not show any association in the univariate analysis, showed a significant association for lower prevalence of CMD in the multivariate analysis. In marital status, only separated individuals showed higher prevalence than single individuals (the reference category) in the multivariate analysis.

There was also significant association of AAD with all
Table 1. Sociodemographic characteristics of the sample $(\mathrm{n}=$ 2921).

\begin{tabular}{|c|c|c|}
\hline Variable & $\mathrm{N}$ & $\%$ \\
\hline \multicolumn{3}{|l|}{ Gender } \\
\hline Male & 1005 & 34.4 \\
\hline Female & 1916 & 65.6 \\
\hline \multicolumn{3}{|l|}{ Ethnicity } \\
\hline White & 1891 & 64.7 \\
\hline Non-white & 1030 & 35.3 \\
\hline \multicolumn{3}{|l|}{ Marital status } \\
\hline Single & 737 & 25.2 \\
\hline Married $^{*}$ & 1761 & 60.3 \\
\hline Separated & 207 & 7.1 \\
\hline Widower/Widow & 216 & 7.4 \\
\hline \multicolumn{3}{|l|}{ Age } \\
\hline $14-19$ years of age & 419 & 14.3 \\
\hline $20-39$ years of age & 1210 & 41.4 \\
\hline $40-49$ years of age & 537 & 18.4 \\
\hline $50-59$ years of age & 407 & 13.9 \\
\hline$>60$ years of age & 348 & 11.9 \\
\hline \multicolumn{3}{|l|}{ Schooling level } \\
\hline Illiterate & 215 & 7.4 \\
\hline Incomplete fundamental level & 1701 & 58.2 \\
\hline Complete fundamental level & 371 & 12.7 \\
\hline Incomplete medium level & 285 & 9.8 \\
\hline Complete medium level & 349 & 11.9 \\
\hline \multicolumn{3}{|l|}{ Employment situation } \\
\hline Employed & 804 & 27.5 \\
\hline Retired senior & 214 & 7.3 \\
\hline Student & 297 & 10.2 \\
\hline Harvest worker & 495 & 16.9 \\
\hline Housewife & 322 & 11 \\
\hline Unemployed & 344 & 11.8 \\
\hline Retired due to disability, health benefit & 361 & 12.4 \\
\hline Did not answer & 84 & 2.9 \\
\hline \multicolumn{3}{|l|}{ Family income } \\
\hline up to $1 \mathrm{MW}^{\dagger}$ & 501 & 17.2 \\
\hline $1-3 \mathrm{MW}$ & 2002 & 68.5 \\
\hline$>3 \mathrm{MW}$ & 418 & 14.3 \\
\hline \multicolumn{3}{|l|}{ SRQ-20 screeening } \\
\hline Yes (score $\geq 8$ ) & 945 & 32.4 \\
\hline No $($ score $<8)$ & 1976 & 67.6 \\
\hline \multicolumn{3}{|l|}{ SRQ-A screening } \\
\hline Yes (score $\geq 2$ ) & 301 & 10.3 \\
\hline No $($ score $<2)$ & 2620 & 89.7 \\
\hline \multicolumn{3}{|l|}{ SRQ-20 and SRQ-A screening } \\
\hline Yes & 1098 & 37.6 \\
\hline No & 1823 & 62.4 \\
\hline
\end{tabular}

SRQ-20 = Self-reporting questionnaire 20 items. SRQ-A = Self-reporting questionnaire for AADD screening. "Married = official union and non-official union of min. one year; ${ }^{\dagger} \mathrm{MW}=$ minimum wage; $\mathrm{R} \$ 30,000$ : three hundred reais or about one hundred dollars when the study was performed. 
Table 2. Prevalence rates of CMD (Common Mental Disorders) suspected cases (using SRQ-20 screening tool) according to demographic variables $(\mathrm{n}=2921)$.

\begin{tabular}{|c|c|c|c|c|}
\hline Variable & Raw PR & $\mathrm{P}$ & Adjusted PR ${ }^{*}$ & $\mathrm{P}$ \\
\hline \multicolumn{5}{|l|}{ Gender } \\
\hline Male & 1 & & 1 & \\
\hline Female & $1.90(1.66-2.18)$ & $<0.001$ & $1.74(1.52-2.00)$ & $<0.001$ \\
\hline \multicolumn{5}{|l|}{ Age } \\
\hline $14-19$ years of age & 1 & & 1 & \\
\hline $20-39$ years of age & $1.20(1.00-1.44)$ & 0.054 & $0.89(0.71-1.12)$ & 0.322 \\
\hline $40-49$ years of age & $1.32(1.07-1.62)$ & 0.008 & $0.90(0.70-1.16)$ & 0.435 \\
\hline $50-59$ years of age & $1.32(1.06-1.64)$ & 0.012 & $0.81(0.62-1.06)$ & 0.121 \\
\hline$>60$ years of age & $0.95(0.74-1.22)$ & 0.672 & $0.65(0.47-0.89)$ & 0.007 \\
\hline \multicolumn{5}{|l|}{ Ethnicity } \\
\hline White & 1 & & - & - \\
\hline Non-white & $1.80(1.05-1.32)$ & 0.004 & - & - \\
\hline \multicolumn{5}{|l|}{ Marital status } \\
\hline Single & 1 & & 1 & \\
\hline Married $^{\dagger}$ & $1.27(1.10-1.47)$ & 0.001 & $1.11(0.94-1.32)$ & 0.202 \\
\hline Separated & $1.80(1.47-2.21)$ & $<0.001$ & $1.27(1.02-1.58)$ & 0.003 \\
\hline Widower/Widow & $1.28(1.00-1.62)$ & 0.046 & $1.02(0.79-1.32)$ & 0.89 \\
\hline \multicolumn{5}{|l|}{ Employment situation } \\
\hline Employed & 1 & & 1 & \\
\hline Retired senior & $0.55(0.36-0.83)$ & 0.004 & $0.62(0.40-0.95)$ & 0.029 \\
\hline Student & $0.97(0.74-1.27)$ & 0.827 & $0.84(0.59-1.19)$ & 0.335 \\
\hline Harvest worker & $1.73(1.44-2.10)$ & $<0.001$ & $1.27(1.05-1.54)$ & 0.014 \\
\hline Housewife & $2.10(1.73-2.55)$ & $<0.001$ & $1.41(1.15-1.72)$ & 0.001 \\
\hline Unemployed & $2.01(1.65-2.44)$ & $<0.001$ & $1.53(1.25-1.87)$ & $<0.001$ \\
\hline Retired due to disability, health benefit & $2.56(2.15-3.06)$ & $<0.001$ & $2.14(1.77-2.59)$ & $<0.001$ \\
\hline Did not answer & $2.33(1.76-3.08)$ & $<0.001$ & $1.72(1.31-2.26)$ & $<0.001$ \\
\hline \multicolumn{5}{|l|}{ Schooling level } \\
\hline Illiterate & $2.60(1.94-3.48)$ & $<0.001$ & $2.03(1.49-2.77)$ & $<0.001$ \\
\hline Incomplete fundamental level & $2.14(1.66-2.75)$ & $<0.001$ & $1.66(1.28-2.14)$ & $<0.001$ \\
\hline Complete fundamental level & $1.74(1.3-2.33)$ & $<0.001$ & $1.43(1.08-1.90)$ & 0.013 \\
\hline Incomplete medium level & $1.25(0.89-1.75)$ & 0.19 & $1.16(0.83-1.61)$ & 0.378 \\
\hline Complete medium level & 1 & & & \\
\hline \multicolumn{5}{|l|}{ Family income } \\
\hline up to $1 \mathrm{MW}^{\ddagger}$ & $2.64(2.07-3.37)$ & $<0.001$ & $1.83(1.43-2.34)$ & $<0.001$ \\
\hline $1-3 \mathrm{MW}$ & $1.87(1.48-2.34)$ & $<0.001$ & $1.50(1.20-1.87)$ & $<0.001$ \\
\hline$>3 \mathrm{MW}$ & 1 & & 1 & \\
\hline
\end{tabular}

$\overline{\mathrm{PR}}=$ prevalence rates weighted for gender. ${ }^{*}$ Used Poisson Regression with roburt variance Method to gender, age, marital status, employment situation, schooling level and family income; ${ }^{\dagger}$ Married = Official union and non-official union of min. one year; ${ }^{\dagger} \mathrm{MW}=\mathrm{Minimum}$ wage; R\$ $30,000=$ three hundred reais or about one hundred dollars when the study was performed. 
Table 3. Prevalence rates of AADD (Alcohol Abuse and Dependence Disorders) suspected cases (using SRQ-A screening tool) according to demographic variables $(\mathrm{n}=2921)$.

\begin{tabular}{|c|c|c|c|c|}
\hline Variable & Raw PR & $\mathrm{P}$ & Adjusted $\mathrm{PR}^{*}$ & $\mathrm{P}$ \\
\hline \multicolumn{5}{|l|}{ Gender } \\
\hline Female & 1 & & 1 & \\
\hline Male & $3.36(2.69-4.19)$ & $<0.001$ & $4.06(3.23-5.10)$ & $<0.001$ \\
\hline \multicolumn{5}{|l|}{ Age } \\
\hline $14-19$ years of age & 1 & & - & - \\
\hline $20-39$ years of age & $2.07(1.35-3.16)$ & 0.001 & - & - \\
\hline $40-49$ years of age & $2.74(1.76-4.28)$ & $<0.001$ & - & - \\
\hline $50-59$ years of age & $2.15(1.32-3.48)$ & 0.002 & - & - \\
\hline$>60$ years of age & $1.56(0.91-2.67)$ & 0.104 & - & - \\
\hline \multicolumn{5}{|l|}{ Ethnicity } \\
\hline White & 1 & & - & - \\
\hline Non-white & $1.37(1.11-1.71)$ & 0.004 & - & - \\
\hline \multicolumn{5}{|l|}{ Marital status } \\
\hline Single & 1 & & - & - \\
\hline Married $^{\dagger}$ & $1.21(0.92-1.58)$ & 0.174 & - & - \\
\hline Separated & $2.12(1.46-3.07)$ & $<0.001$ & - & - \\
\hline Widower/Widow & $1.05(0.64-1.74)$ & 0.842 & - & - \\
\hline \multicolumn{5}{|l|}{ Employment situation } \\
\hline Employed & 1 & & 1 & \\
\hline Retired seniors & $0.69(0.40-1.19)$ & 0.182 & $0.83(0.49-1.43)$ & 0.509 \\
\hline Student & $0.31(0.16-0.58)$ & $<0.001$ & $0.40(0.22-0.76)$ & 0.005 \\
\hline Harvest worker & $1.04(0.75-1.45)$ & 0.807 & $1.35(0.99-1.86)$ & 0.006 \\
\hline Housewife & $0.56(0.34-0.91)$ & 0.002 & $1.07(0.65-1.75)$ & 0.796 \\
\hline Unemployed & $1.97(1.47-2.64)$ & $<0.001$ & $1.98(1.49-2.64)$ & $<0.001$ \\
\hline Retired due to disability, health benefit & $1.39(1.00-1.94)$ & 0.049 & $1.32(0.95-1.83)$ & 0.102 \\
\hline Did not answer & $0.55(0.21-1.47)$ & 0.232 & $0.71(0.27-1.85)$ & 0.48 \\
\hline \multicolumn{5}{|l|}{ Schooling level } \\
\hline Illiterate & $1.88(1.07-3.27)$ & 0.027 & - & - \\
\hline Incomplete fundamental level & $1.89(1.21-2.95)$ & 0.005 & - & - \\
\hline Complete fundamental level & $1.56(0.92-2.64)$ & 0.099 & - & - \\
\hline Incomplete medium level & $0.84(0.45-1.58)$ & 0.595 & - & - \\
\hline Complete medium level & & & - & - \\
\hline \multicolumn{5}{|l|}{ Family income } \\
\hline up to $1 \mathrm{MW}^{\ddagger}$ & $2.12(1.45-3.1)$ & $<0.001$ & - & - \\
\hline $1-3 \mathrm{MW}$ & $1.15(0.80-1.64)$ & 0.444 & - & - \\
\hline$>3 \mathrm{MW}$ & 1 & & - & - \\
\hline \multicolumn{5}{|l|}{ SRQ - 20} \\
\hline Positive (score $\geq 8$ ) & $1.96(1.59-2.43)$ & $<0.001$ & $2.31(1.87-2.86)$ & $<0.001$ \\
\hline Negative ( score < 8 ) & 1 & & 1 & \\
\hline
\end{tabular}

$\mathrm{PR}=$ prevalence rates weighted for gender. ${ }^{*}$ Used Poisson Regression Method with robust variance to gender, employment situation and screening with SRQ-20 (Self-reporting Questionnaire 20 items); ${ }^{\dagger}$ Married = Official union and non-official union of min. one year; ${ }^{\dagger} \mathrm{SW}=\mathrm{Minimum}$ wage; R $\$ 30,000=$ three hundred reais when the study was performed. 
sociodemographic variables and suspected cases of CMD in the univariate analysis (Table 3). All of them were included in the first stage of the multivariate analysis: age, ethnicity, marital status, schooling and income did not show significance and therefore were not considered in the following stage.

As Table 3 shows, gender and CMD presented the same standard of association in both multivariate and univariate analyses; that is, higher PR for males and for individuals with positive screening for CMD. For employment, the housewife and retired due to disabilityhealth benefit categories lost the significance in the multivariate analysis, while student, harvest worker and unemployed still showed significance.

\section{DISCUSSION}

\subsection{Main Findings}

The main findings of this study are the recognition of population segment at higher risk for $\mathrm{CMD}$ and $\mathrm{AAD}$ and the independent association of CMD and AAD with unfavorable sociodemographic indicators.

In the univariate analysis, prevalence estimates of suspected cases of CMD were significantly higher in women, adults between 20 and 59 years of age, nonwhite, individuals with unfavorable employment situation, low schooling level and low family income. Female, unfavorable employment condition, low schooling level and low income showed positive and independent associations with CMD, that is, the associations remained after multivariate adjustments.

The association of females and CMD has been observed since the first population-based studies in psychiatry [10-18] and all psychiatrists are aware of this finding. Although it has been suggested several biological (e.g., hormonal) and social (e.g., cultural role) susceptibility mechanisms in women for these pathologies, there is no any certainty yet.

Unfavorable socioeconomic conditions (unfavorable employment condition, low schooling level and low income) showed independent association with CMD. It is in accordance with other studies [10-18]. The reason for these associations is merely an area of speculation: it can be a cause or/and a consequence of mental disorders. Financial strain of unemployment can lead to low social status and low self-esteem, which can increase the risk of mental illness [19]. The 2004-2005 Wave 2 National Epidemiologic Survey on Alcohol and Related Conditions (NESARC), based on the 3-year prospective followup of the Wave 1 NESARC (2001-2002), presented U.S. representative findings on sociodemographic predictors of first incidence of DSM-IV substance, mood and anxiety disorders. The total sample was composed by 34,653 subjects. Low family income was significantly related to risks of most clinical manifested anxiety and depressive disorders [18].

On the other hand, we have to consider clinical and epidemiological evidences that sub-syndromic psychiatric symptoms (sometimes beginning in the childhood) could be precipitators of social stressors that would end up favoring the symptoms and the complete clinical expression of mental disorders. This proposal has been demonstrated in longitudinal studies about bipolar disorder that have addressed both factors in an instigating fashion [20,21].

These associations also reflect the methodological problems that require the study about these relations. In this case, the similarity of schooling level, employment situation and income associations with CMD show that there is an interaction with one another and reflect the study complexity involving socioeconomic variables due to their interdependence. It is incontestable that the employment situation affects income, as schooling level affects the employment situation.

It is interesting to note that the harvest worker category presented an association with CMD with intermediate PR between employed and unemployed individuals. Indeed, this is an intermediate category involving employed and unemployed people, since they work during the harvest period of tobacco (a typical culture in the region) and are unemployed in the rest of the year, as they do not receive salary and have no guarantee of work in the following period. This pattern of association shows a dose-effect response.

The association of age with CMD is controversial in literature, ranging from a direct linear correlation to nonassociation [10-18]. Our study showed higher PRs for adults between 20 and 59 years of age, similar of other recent studies [15-18]. We observed reduced PRs for all categories in the multivariate analysis when compared to those from the univariate analysis. Individuals over 60 years of age presented prevalence significantly lower than the reference category after the multivariate adjustment. The National Comorbidity Survey Replication in the United States showed this type of independent association found here with age [22].

The association of marital status with CMD is also controversial. In our study, married and divorced/widow individuals presented higher prevalence estimates of suspected cases of CMD when compared to the reference category (single) in the univariate analysis; in the multivariate analysis, the difference in prevalence estimates was obtained only for divorced/widow category.

In relation to $\mathrm{AAD}$, in the univariate analysis we observed significantly higher prevalence estimates of suspected cases in men, adults between 20 and 59 years of age, non-white, separated, unfavorable employment situation, low schooling level, lower family income and 
suspected cases of CMD. The variables that presented association with $\mathrm{AAD}$ after the multivariate analysis were: gender, employment situation and CMD. The standard association of AAD with sociodemographic variables is not very consistent with other studies. The exception is male variable, which is associated with AAD in all studies [15-18,22-25]. This association is also very well-established in psychiatric epidemiology, like the association between females and CMD.

The analysis that considered AAD as outcome included CMD in the predictor variables because several studies have demonstrated a consistent relation with CMD in individuals with AAD, as well as high frequency of CMD in individuals with AAD. It is possible that CMD predisposes to AAD. The association of AAD with CMD, observed in several studies [14-18], including this one, combined with the high prevalence of CMD in individuals with AAD (45.62\%; IC 95\% 43.66\% $47.59 \%$ ), reinforce this idea of association between CMD and AAD.

Despite the fact it is not a random sample we briefly discuss the prevalence estimates of CMD and AAD in our study: $29.93 \%$ (IC 95\% 29.30\% - 30.56\%) and $12.07 \%$ (IC 95\% 11.63\% - 12.52\%), respectively. In the literature, there are four Brazilian population-based studies that used SRQ-20 as screening instrument. Prevalence estimates found were: $35 \%$ in Olinda and $36 \%$ in Zona da Mata, both in Pernambuco [10,11], 28.5\% in Pelotas [12] and 24.1\% in São Paulo [13]. Four population-based studies conducted in Brazil showed prevalence estimates of $\mathrm{AAD}$ ranging from $6.2 \%$ to $8 \%$ [5, 23-25].

\subsection{Limitations}

The main limitations of this study are the reduced generalization capability to populations of higher variability in sociodemographic indicators and the high rate of refusal $(46.42 \%)$, although the latter was attenuated by the large sample that might minimize random errors. However, it does not prevent the possibility of higher participation of cases or non-cases, which could change the prevalence estimates, but does not change the association patterns found. One of the reasons for the refusals could be the fact that the CHWs, residents of the studied area, were the researchers of the study. In spite of the efforts to keep the confidentiality of the collected data, some of the eligible sample may have felt embarrassed in participating.

The associations observed in cross-sectional studies like this one are not always related to the prevalence of the disease but the duration of the disease. Therefore, the associations of this study could be partially related to the duration of the mental disorders. For example, low socioeconomic status could result on no access to a satisfac- tory treatment in order to prolong the duration of the disorders. That is, unfavorable social events are simultaneously the cause and consequence of mental disorders as well as the contribution factor for the progression of the disorder, establishing a "vicious circle".

\section{CONCLUSIONS AND IMPLICATIONS}

The design of this study (cross-sectional) does not show temporal relationship for the associations we found here, essential for causality. However, we will discuss some relevant issues. Theories derived from social psychiatry, such as stress-producing life events, low social support, difficulties in performing social roles and loss of own life control sensation, have been discussed as counterpoints to the biological model for the etiology of mental disorders $[14,26]$.

Unfortunately, these theories do not value the individual susceptibility in terms of genetics expressed through characteristics of temperament (personality component of biological determination in opposition to the character, personality component of predominantly environmental determination) for the occurrence of these disorders. The dialog among social, clinical and experimental psychiatry seems to be important. This interface may bring important contributions to boost progress in the area of causality.

Considering both biological and social phenomena in the genesis of mental disorders is important, as they seem to contribute to the web causes. Today, the tendency is to consider the biological factors as primary cause and the environmental factors as mediating factors [27]. Mediating factors are those situated between exposure and outcome, favoring the occurrence of the latter. However, they do not need to be present for the exposure to cause the outcome, as the association of cause and effect occurs by adding up the direct and indirect effects. This way, eliminating the mediating factor does not completely prevent the outcome, but it is an important focus for interventions to reduce the outcome occurrence. The importance of these mediating factors has been explored lately and their function has been better outlined mainly in the mental health area [28].

The diathesis-stress model for mental disorders proposes that unfavorable conditions of the environment interacting with the individual biological susceptibility to the disease end up in clinical manifestation or aggravation. We suggest widening of what is considered as the environmental conditions of the model. Instead of being limited to lifestyle or person-to-person close interactions (with parents, friends, colleagues), it seems important to include the socioeconomic and social conditions in general, which many times have been unconsidered as a risk factor for the disease. This way, interventions in indicators of unfavorable socioeconomic conditions could 
be an important point of intervention.

Finally, the evidences show the best way to widen the assistance in mental disorders is to implement public policies for the area at the primary care level. This is especially important when we consider the risk groups for mental disorders are the most vulnerable in terms of socioeconomic status. These groups face the greatest obstacles in finding proper health assistance.

Those public policies could result in early diagnoses and in effective treatments, attenuating the impact of mental disorders at any level. From an administrative point of view, such policies could represent benefits because it is possible to use the infra-structure already in place, which will result in a wider and cost-efficient assistance in mental health. In Brazil, this recommenddation implies necessarily that the ESF teams, considered as the reorganization strategy of the primary health care in this country, are actively engaged in the process.

\section{REFERENCES}

[1] WHO (2001) The World Health Report 2001: Mental health new understanding, new hope. World Health Organization, Geneva.

[2] WHO (2004) The treatment gap in mental health care. Bulletin of the World Health Organization, 82.

[3] Grandes, G., Montoya, I., Arietaleanizbeaskoa, M.S., Arce, V. and Sanchez, A. (2011) The burden of mental disorders in primary care. European Psychiatry: The Journal of the Association of European Psychiatrists, 26, 428-435. doi:10.1016/j.eurpsy.2010.11.002

[4] Pereira, M.G. (2001) Indicadores de saúde [health indicators]. In: Pereira, M.G., Epidemiologia: Teoria e prática [Epidemiology: Theory and Practice], GuanabaraKoogan, Rio de Janeiro, 49-73.

[5] Barros, M.B.A., Botega, N.J., Dalgalarrondo, P., MarínLeón, L. and Oliveira, H.B. (2007) Prevalence of alcohol abuse and associated factors in a population-based study. Revista de Saúde Pública, 41, 502-509. doi:10.1590/S0034-89102006005000032

[6] Ministério da Saúde, Informações de Saúde_-Estatísticas Vitais. Sistema de informações sobre mortaliade/MS/ SUS/DASIS. http://tabnet.datasus.gov.br

[7] Harding, T.W., de Arango, M.V., Baltazar, J., Climent, C.E., Ibrahim, H.H., Ladrido-Ignacio, L., Murthy, R.S. and Wig, N.N. (1980) Mental disorders in primary health care: A study of their frequency and diagnosis in four developing countries. Psychological Medicine, 10, 231-241. doi:10.1017/S0033291700043993

[8] Climent, C.E. and De Arango, M.V. (1983) Manual de Psiquiatria para trabajadores de atencion primaria [Psychiatry Manual to primary care workers]. Organización Panamericana de la Salud [Pan American Health Organization], Washington DC.

[9] Gonçalves, D.M., Stein, A.T. and Kapczinski, F. (2008) Avaliação de desempenho do self-report questionnaire como instrumento de rastreamento psiquiátrico: Um estudo comparativo com o structured clinical interview for DSM-IV-TR [performance of the self-reporting questionnaire as a psychiatric screening questionnaire: A comparative study with structured clinical interview for DSM-IV-TR]. Cadernos de Saúde Pública, 24, 380-390. doi:10.1590/S0102-311X2008000200017

[10] Costa, A.G. and Ludermir, A.B. (2005) Transtornos mentais comuns e apoio social: Estudo em comunidade rural da Zona da Mata de Pernambuco, Brasil [Common mental disorders and social support in a rural community in Zona da Mata, Pernambuco State, Brazil]. Cadernos de Saúde Pública, 21, 73-79. doi:10.1590/S0102-311X2005000100009

[11] Ludermir, A.B. and Melo Filho, D.A. (2002) Condições de vida e estrutura ocupacional associadas a transtornos mentais comuns [Living conditions and occupational organization associated with common mental disorders]. Revista de Saúde Pública, 36, 213-221. doi:10.1590/S0034-89102002000200014

[12] Costa, J.S.D., Menezes, A.M.B., Olinto, M.T.A., Gigante, D.P., Macedo, S. and Britto, M.A.P. (2002) Prevalência de distúrbios psiquiátricos menores na cidade de Pelotas, RS [Prevalence of minor psychiatric disorders in Peltas, RS]. Revista Brasileira de Epidemiologia, 5, 164-173. doi:10.1590/S1415-790X2002000200004

[13] Maragno, L., Goldbaum, M., Gianini, R.J., Novaes, H.M.D. and César, C.L.G. (2006) Prevalência de transtornos mentais comuns em populações atendidas pelo Pro- grama de Saúde da Família (QUALIS) no Município de São Paulo, Brasil [Prevalence of common mental disorders in a population covered by the Family Health Program (QUALIS) in São Paulo, Brazil]. Cadernos de Saúde Pública, 22, 1639-1648. doi:10.1590/S0102-311X2006000800012

[14] Almeida-Filho, N., Mari, J.J., Coutinho, E., Franca, J.F., Fernandes, J., Andreoli, S.B. and Busnello, E.D. (1997) Brazilian multicentric study of psychiatric morbidity. Methodological features and prevalence estimates. The British Journal of Psychiatry: The Journal of Mental Science, 171, 525-529.

[15] Goodman, A.B., Siegel, C., Craig, T.J. and Lin, S.P. (1983) The relationship between socioeconomic class and prevalence of schizophrenia, alcoholism, and affective disorders treated by inpatient care in a suburban area. The American Journal of Psychiatry, 140, 166-170.

[16] Williams, D.R., Herman, A., Stein, D.J., Heeringa, S.G., Jackson, P.B., Moomal, H. and Kessler, R.C. (2008) Twelve-month mental disorders in South Africa: Prevalence, service use and demographic correlates in the population-based South African Stress and Health Study. Psychological Medicine, 38, 211-220. doi: $10.1017 / \mathrm{S} 0033291707001420$

[17] Chong, S.A., Abdin, E., Vaingankar, J.A., Heng, D., Sherbourne, C., Yap, M., Lim, Y.W., Wong, H.B., GhoshDastidar, B., Kwok, K.W. and Subramaniam, M. (2012) A population-based survey of mental disorders in Singapore. Annals of the Academy of Medicine, 41, 49- 66.

[18] Grant, B.F., Goldstein, R.B., Chou, S.P., Huang, B., Stinson, F.S., Dawson, D.A., Saha, T.D., Smith, S.M., Pulay, 
A.J., Pickering, R.P., Ruan, W.J. and Compton, W.M. (2009) Sociodemographic and psychopathologic predictors of first incidence of DSM-IV substance use, mood, and anxiety disorders: Results from the wave 2 national epidemiologic survey on alcohol and related conditions. Molecular Psychiatry, 14, 1051-1066. doi:10.1038/mp.2008.41

[19] Rodríguez, E., Frongillo, E.A. and Chandra, P. (2001) Do social programmes contribute to mental well-being? The long-term impact of unemployment on depression in the United States. International Journal of Epidemiology, 30, 163-170. doi:10.1093/ije/30.1.163

[20] Murphy, J.M., Olivier, D.C., Monson, R.R., Sobol, A.M., Federman, E.B. and Leighton, A.H. (1991) Depression and anxiety in relation to social status. Archives of General Psychiatry, 48, 223-229. doi:10.1001/archpsyc.1991.01810270035004

[21] Reilly-Harrington, N.A., Alloy, L.B., Fresco, D.M. and Whitehouse, W.G. (1999) Cognitive styles and life events interact to predict bipolar and unipolar symptomatology. Journal of Abnormal Psychology, 108, 567-578. doi:10.1037/0021-843X.108.4.567

[22] Kessler, R.C., Chiu, W.T., Demler, O. and Walters, E.E. (2005) Prevalence, severity, and comorbidity of 12-month DSM-IV disorders in the national comorbidity survey replication. Archives of General Psychiatry, 62, 617-627.

\section{doi:10.1001/archpsyc.62.6.617}

[23] Galduroz, J.C., Noto, A.R., Nappo, S.A. and Carlini, E.L. (2003) First household survey on drug abuse in São Paulo, Brazil, 1999: Principal findings. São Paulo Medical Journal, 121, 231-237. doi:10.1590/S1516-31802003000600003

[24] Mendoza-Sassi, R.A. and Beria, J.U. (2003) Prevalence of alcohol use disorders and associated factors: A population-based study using AUDIT in Southern Brazil. Addiction, 98, 799-804. doi:10.1046/j.1360-0443.2003.00411.x

[25] Almeida-Filho, N., Lessa, I., Magalhães, L., Araújo, J.M., Aquino, E., James, S.A. and Kawachi, I. (2005) Social inequality and alcohol consumption-abuse in Bahia, Brazil. Social Psychiatry and Psychiatric Epidemiology, 40, 214-222. doi:10.1007/s00127-005-0883-4

[26] Cassell, J. (1974) Psychosocial processes and stress: Theoretical formulation. International Journal of Health Services, 4, 471-482. doi:10.2190/WF7X-Y1L0-BFKH-9QU2

[27] MacKinnon, D.P., Krull, J.L. and Lockwood, C.M. (2000) Equivalence of the Mediation, confounding and suppression effect. Prevention Science, 1, 173-181. doi:10.1023/A:1026595011371

[28] Cloninger, C.R. (2004) Feeling good. The Science of Well-Being, Oxford University Press, New York. 